\title{
Integrable models of quantum optics
}

\author{
Vladimir Yudson ${ }^{1,2, *}$ and Aleksander Makarov ${ }^{2,3}$ \\ ${ }^{1}$ National Research Institute Higher School of Economy, 20 Myasnitskaya Str., Moscow, 101000 \\ Russia \\ ${ }^{2}$ Institute of Spectroscopy RAS, 5 Fizicheskaya Str., Troitsk, Moscow 142190 Russia \\ ${ }^{3}$ Moscow Institute of Physics and Technology, 9 Institutskiy Pereulok, Dolgoprudny, 141700 Russia
}

\begin{abstract}
We give an overview of exactly solvable many-body models of quantum optics. Among them is a system of two-level atoms which interact with photons propagating in a one-dimensional (1D) chiral waveguide; exact eigenstates of this system can be explicitly constructed. This approach is used also for a system of closely located atoms in the usual (non-chiral) waveguide or in 3D space. Moreover, it is shown that for an arbitrary atomic system with a cascade spontaneous radiative decay, the fluorescence spectrum can be described by an exact analytic expression which accounts for interference of emitted photons. Open questions related with broken integrability are discussed.
\end{abstract}

The simplest model of quantum optics - a two-level atom interacting with a single mode quantum electromagnetic field (Janes-Cummings model [1]) allows one to find exact states of the system and to describe its dynamics. For a multimode field the problem becomes nontrivial. Examples of a multimode field are a field propagating in a long waveguide, a field in an open 3D space, etc. Solution of the Schrödinger equation for a multi-atom - multiphoton system is not possible in general. However, this is possible for some particular models of quantum optics which we describe below.

1. A system of two-level atoms in a 1D waveguide with chiral photons described by the Hamiltonian (a quantum generalization of the classical model)

$$
H=-i \int d x a^{+}(x) \partial_{x} a(x)-\sqrt{\gamma} \sum_{j}\left[s_{j}^{+} a\left(x_{j}\right)+s_{j}^{-} a^{+}\left(x_{j}\right)\right],
$$

where $a(x)$ is the photon annihilation operator at point $x$ and spin-1/2 operator $\vec{S}_{j}$ describes a two-level atom located at the point $x_{j}$. The exact eigenstates of this Hamiltonian for an arbitrary number of photons and excited atoms were constructed in [3] with the use of Bethe Ansatz [4]. The chirality (unidirectional propagation) of photons is the key condition for the integrability of this model.

2. A system of M identical two-level atoms located in a spatial region (of a size small as compared with the resonant wavelength) and interacting with electromagnetic field in 3D space (Dicke model [5]). As shown in [6], this 3D model can be mapped on an effective 1D

" Corresponding author: yudson@isan.troitsk.ru 
model Eq. (1), but with chiral photons interacting with a spin $\mathrm{M} / 2$ located at the point $x=0$. Such a model also allows one to construct exact multi-photon eigenstates [6]. Knowledge of the full set of eigenstates and an efficient method developed in [7] for summation over so called string configurations (correlated packets of several photons) allows one to describe dynamics of an arbitrary initial state of the many-body atom-photon system. In particular, it has become possible to describe not only the cascade decay dynamics of the atomic system down to the ladder of Dicke's states, but also to describe the time evolution of multiphoton functions and to calculate the superfluorescence spectrum.

3. Exactly, albeit in a more cumbersome way, one can solve also a problem of $\mathrm{N}$ photons interacting with an ensemble of $\mathrm{M}$ closely located two-level atoms in the usual (non-chiral) $1 \mathrm{D}$ waveguide [8]. This is possible because there are no photon returns to the atomic system in this model. Such returns are present already in a simplest system of separated atoms in the non-chiral waveguide; they essentially influence on the system dynamics and, for instance, lead to existence of dark states [9].

4. An exact description is possible also for an arbitrary atomic system with a cascade system of optical transitions. The absence of branching and returns allows one to find the exact solution of the Schrödinger equation for the amplitudes of the states with arbitrary numbers of emitted photons. In this way one can obtain a general expression for the spectrum of the cascade emission [10]:

$$
S(\omega)=\frac{1}{2 \pi i} \sum_{l=1}^{N} \frac{1}{\Gamma_{l, l-1}} \sum_{k=1}^{l} \prod_{j=k}^{l} \frac{\Gamma_{j, j-1}}{\omega-\left(E_{j}-E_{j-1}\right)-\frac{i}{2}\left(\Gamma_{j, j-1}+\Gamma_{j-1, j-2}\right)}+c . c .
$$

where $E_{j}$ is the energy of the state $|j\rangle, \Gamma_{j, j-1}$ - the rate of the transition $|j\rangle \rightarrow|j-1\rangle$. This expression accounts also for the case of close frequencies of some transitions (so that the emitted photon can interfere). For a particular case of the cascade emission in the Dicke model, Eq. (2) coincides with that calculated in [6] with the use of Bethe Ansatz; the transition rates are $\Gamma_{j, j-1}=j(N-j+1) \Gamma$.

\section{References}

[1] E.T. Jaynes, F.W. Cummings, Proc. IEEE 51, 89 (1963)

[2] J.C. MacGillivray and M.S. Feld, Phys. Rev. A 14, 1169 (1976)

[3] V.I. Rupasov, V.I. Yudson, Sov. Phys. JETP 59(3), 478 (1984)

[4] H. Bethe, Z. Phys. 71, 205 (1931)

[5] R.H. Dicke, Phys. Rev. 93, 99 (1954)

[6] V.I. Rupasov, V.I. Yudson, Sov. Phys. JETP 60, 927 (1984)

[7] V.I. Yudson, Sov. Phys. JETP 61, 1043 (1985)

[8] V.I. Yudson, P. Reineker, Phys.Rev. A 78, 052713 (2008)

[9] A.A. Makarov, V.S. Letokhov, JETP 97, 688 (2003)

[10] A.A. Makarov, V.I. Yudson, Phys. Rev. A 89, 053806 (2014) 